\title{
Tratamiento farmacológico de la depresión en el embarazo
}

\author{
Pharmacologic treatment of depression during pregnancy. \\ Wisner K., Gelemberg A., Leonard H. et al. JAMA 1999 0ct 6; 282: 1264-69
}

\section{Objetivo}

Identificar riesgos asociados con el tratamiento de la depresión mayor durante el embarazo.

\section{Fuentes de datos}

Se identificaron estudios prospectivos y controlados en inglés realizados entre 1989 y 1999, mediante búsquedas electrónicas (Medline y Health Star), revisión manual de bibliografía y discusión con investigadores del tema.

\section{Selección de datos}

Se seleccionaron estudios donde la exposición a antidepresivos fuera comparada con exposiciones no teratógenicas. Se incluyeron 4 estudios publicados desde 1993.

\section{Extracción de datos}

Se obtuvo información sobre casos, grupos de comparación, complicaciones del embarazo y características de los recién nacidos. Los datos se agruparon según el tipo de toxicidad: 1 . Muerte fetal intrauterina, 2.Teratogenicidad morfológica, 3.Alteraciones del crecimiento, 4. Alteraciones del comportamiento y 5.Toxicidad neonatal.

\section{Resultados principales}

Los antidepresivos evaluados fueron: tricíclicos, fluoxetina y nuevos inhibidores de la recaptación de serotonina (SSRIs): sertralina, paroxetina y fluvoxamina. En tres estudios se compararon tiempos de exposición. Los hallazgos se agruparon en 5 dominios:

1. Muerte intrauterina: no hubo aumento de riesgo con ninguno de los antidepresivos mencionados.
2. Teratogenicidad Morfológica: no se detectó aumento de la tasa de defectos congénitos mayores.

En uno de los 3 estudios con fluoxetina (Chambers y cols) los expuestos a ésta tenían mayor frecuencia de 30 más anormalidades menores, sin repercusión cosmética ni funcional, (15.5\% vs $6.5 \%$ en controles $p=0.03$ )

3. Alteraciones en el crecimiento: sólo en el estudio de Chambers se detectaron diferencias. Los nacidos a término tenían menor talla y peso al nacer y el peso materno era $3 \mathrm{~kg}$ menor en el grupo expuesto a fluoxetina luego de la semana 25 de gestación.

4. Alteraciones del comportamiento: el único estudio que exploró este dominio no encontró diferencias en funciones cognitivas, temperamento y comportamiento general en niños expuestos en etapa prenatal a tricíclicos y fluoxetina.

5. Toxicidad neonatal: sólo el estudio de Chambers detectó diferencias. La admisión a cuidados especiales neonatales fué $23 \%$ en el grupo de exposición tardía a fluoxetina, $9.5 \%$ en el grupo de exposición temprana (antes de la semana 25 de gestación) y $6.3 \%$ en controles $(p=0.001)$. Se detectó pobre adaptación neonatal ( inquietud, hipoglucemia, hipotermia, disminución del tono muscular, distress respiratorio, debilidad del llanto, desaturación durante la alimentación) en 31.5\% de exposiciones tardías y $8.9 \%$ de exposiciones tempranas.

\section{Conclusiones}

A pesar del escaso número de trabajos, la evidencia de estudios prospectivos controlados representa un paso adelante con respecto a estudios previos. Sin embargo la información respecto a fluoxetina es conflictiva y falta información sobre nuevos antidepresivos.

\section{COMENTARIO}

La prevalencia de depresión durante el emabarazo ( 9 a $16 \% 1$ ) es similar a la de mujeres no embarazadas ${ }^{2}$ siendo el primer trimestre la etapa de mayor riesgo. ${ }^{1-2}$

El tratamiento con antidepresivos en este período, requiere una cuidadosa evaluación de riesgos y beneficios.

Esta revisión no sistemática es un aporte interesante aunque insuficiente. Analiza pocos trabajos, no homogéneos y escritos en inglés lo cual acota la obtención de información en un área poco investigada, sin embargo ayuda a aclarar numerosos interrogantes.

Ninguno de los trabajos muestra aumento del riesgo de malformaciones mayores ni abortos por el uso de tricíclicos o SSRIs.

Aunque Chambers refiere aumento de malformaciones menores por fluoxetina, éstas no tienen patrón especifico y sus resultados son opuestos a los de Pastuszak.

En cuanto a la adaptación neonatal, hay evidencia de efectos anticolinérgicos en neonatos expuestos a tricíclicos al final del embarazo? Chambers observó que la fluoxetina empleada en el tercer trimestre resultó en mayores tasas de prematuridad (RR 4.8 IC 95\% 1.1 a 20.8), admisiones a cuidados especiales neonatales (RR 2.6 IC 95\% 1.1 a 6.9), pobre adaptación neonatal (RR 8.7 IC $95 \% 2.9$ a 26.6) y menor peso (188 gr IC $95 \% 32$ a $344 \mathrm{p}<0.02$ ) y talla al nacer. Este estudio compara mujeres deprimidas con controles no deprimidos haciéndose difícil deslindar el efecto de la depresión en sí sobre los resultados. Existe bibliografía sobre asociación de depresión con retardo del crecimiento fetal ${ }^{3}$ y menor control médico del embarazo ${ }^{3}$. Además en embarazadas deprimidas se demostró mayor número de embarazos ${ }^{4-5-6}$ y abortos terapeuticos previos ${ }^{4-5-6}$, mayor consumo de tabaco ${ }^{4-5-6-7-8}$, alcohol ${ }^{6-8}$ y cocaína ${ }^{8}$ y menor nivel socioeconómico ${ }^{5}$.

El riesgo de suicidio en este príodo es idéntico al de mujeres deprimidas no embarazadas ${ }^{1} y$ debe ser siempre considerado en la toma de decisiones. Resumiendo, aunque se necesita más investigación, hay estudios que avalan el uso de tricíclicos disminuyendo la dosis o suspendiendo su uso al final del embarazo. Los SSRIs, las drogas más utilizadas en la actualidad $^{1}$, aparentemente no producen malformaciones mayores ni abortos aunque persisten dudas respecto a potenciales efectos adversos en la adaptación neonatal de los expuestos tardíamente a fluoxetina.

2. De Swiet M. Medical Disorder in Obstetric Practice.1995 3 Ed

3. Coverdale J, Chevernak F, McCullogh L et al. Ethically justified clinically comprehensive guidelines for the management of the depressed pregnant patient. Am J 0bstet Gynecol 1996 jan ; 174: 169-173

4. Chambers Ch, Johnson K, Dick L Birth outcome in pregnant women taking fluoxetine. N Engl J Med. $1996 ; 335: 1010-15$

5. Kulin NA, Pastuszak A, Sage SR Pregnancy outcome following maternal use of the new selective serotonin reuptake inhibitors. JAMA 1998;279: 609-10

6. Nulman I, Rovert J, Stewart DE. Neurodevelopmentof children exposed in utero to antidepressant drugs. N Engl J Med. 1997; 336: 258-62

7. Glassman AH Cigarette Smoking: implications for psychiatric illness. Am J Psichiatry 1993; 150: 546-53

8. Zucherman B, Amaro H, Baucner H. Depressive symptoms during pregnancy: relationship to poor healths behaviors. Am J 0bstet Gynecol 1989;160:1107-11 